\title{
Severe desaturation while attempting one-lung ventilation for congenital cystic adenomatoid malformation with respiratory distress syndrome in neonate
} -A case report-

\author{
Ji-Hye Seok, Eun-Ju Kim, Jong-Seouk Ban, Sang-Gon Lee, Ji-Hyang Lee, Da-Mi Seo, and Kwang-Seok Shim \\ Department of Anesthesiology and Pain Medicine, Daegu Fatima Hospital, Daegu, Korea
}

There are many methods for achieving one-lung ventilation (OLV) during thoracic surgery in neonates and the accuracy of OLV may affect postoperative outcome. The authors have performed OLV using a $5 \mathrm{Fr}$ Arndt endobronchial blocker (AEB, Cook Inc., Bloomington, IN, USA) on a neonate diagnosed with congenital cystic adenomatoid malformation and respiratory distress syndrome (RDS) associated with marked mediastinal shift. In spite of sufficient preoxygenation, sudden and severe fall in oxygen saturation had occurred. Since neonates with RDS may develop sudden and severe desaturation, rapid intubation with anticipation of potential difficulty is necessary as well as sufficient preoxygenation. (Korean J Anesthesiol 2013; 65: 80-84)

Key Words: Congenital cystic adenomatoid malformation, Hypoxia, Newborn, One lung ventilation, Respiratory distress syndrome.

Recently, there has been a gradually increasing trend for thoracic surgery in neonates and infants [1], and in such cases the surgical approach can be more convenient through one-lung ventilation (OLV) [2]. Broadly, there are two methods of OLV in neonates, a deliberate endobronchial intubation of a singlelumen endotracheal tube (ETT) and the use of a bronchial blocker. The former is not a technically difficult procedure but has several shortcomings, such as the incomplete collapse of the operating lung; contamination from the affected lung to the healthy lung; difficulties in suctioning of the lung during operation; and the possibility of obstruction of the upper lobe bronchus (especially the right upper lobe bronchus) [3].

AEB, a type of bronchial blocker, is widely used in neonates and infants as well as adults for having advantageous properties such as a high-volume, low-pressure balloon and a multiport airway adapter [4-6].

There are, to our knowledge, no reports regarding OLV of neonates in South Korea, and very few reports worldwide regarding OLV especially in a neonate with accompanying respiratory distress.

The authors experienced a rapidly progressing decline in

Received: August 8, 2012. Revised: 1st, August 24, 2012; 2nd, September 4, 2012. Accepted: September 5, 2012.

Corresponding author: Eun-Ju Kim, M.D., Department of Anesthesiology and Pain Medicine, Daegu Fatima Hospital, 576-31, Sinam-dong, Dong-gu, Daegu 701-600, Korea. Tel: 82-53-940-7436, Fax: 82-53-954-7417, E-mail: kej1127@fatima.or.kr

(c) This is an open-access article distributed under the terms of the Creative Commons Attribution Non-Commercial License (http:// creativecommons.org/licenses/by-nc/3.0/), which permits unrestricted non-commercial use, distribution, and reproduction in any medium, provided the original work is properly cited. 
oxygen saturation while attempting endotracheal intubation for OLV in a neonate with congenital cystic adenomatoid malformation (CCAM) and respiratory distress syndrome (RDS). Through a prompt management, we were successful in oxygenation and OLV, and thus we report the case.

\section{Case Report}

A female neonate with a gestational age of 38 weeks and weight of 3,600 g who was born through a normal vaginal delivery was transferred to our hospital due to cyanosis, tachypnea of more than 70 breaths per minute, and chest retraction during respiration directly after birth. Even after administering $1 \mathrm{~L} / \mathrm{min}$ of oxygen through a nasal cannula, the oxygen saturation remained at less than $90 \%$. Hence, an ETT with an internal diameter of $4.5 \mathrm{~mm}$ was intubated, and ventilator care was performed. With high frequency oscillation ventilation (HFOV) of $10 \mathrm{~Hz}$ and fraction of inspired oxygen $\left(\mathrm{FiO}_{2}\right)$ of 0.5 , oxygen saturation was maintained at 99$100 \%$. Afterward, the ventilator was switched to synchronized intermittent mandatory ventilation (SIMV) mode and weaning was attempted, but was unsuccessful as the neonate's spontaneous breathing was weak.

On the chest CT performed at our hospital, cystic lesions in the right lower lobe, subsequent left mediastinal shift from the lesions, and atelectasis in the left upper lobe were discovered (Fig. 1, 2). She was diagnosed with type II CCAM and RDS grade III, and a right lower lobectomy was scheduled at 4 days after birth. A $3.1 \mathrm{~mm}$ fiberoptic bronchoscopy (FOB) (LF-DP,

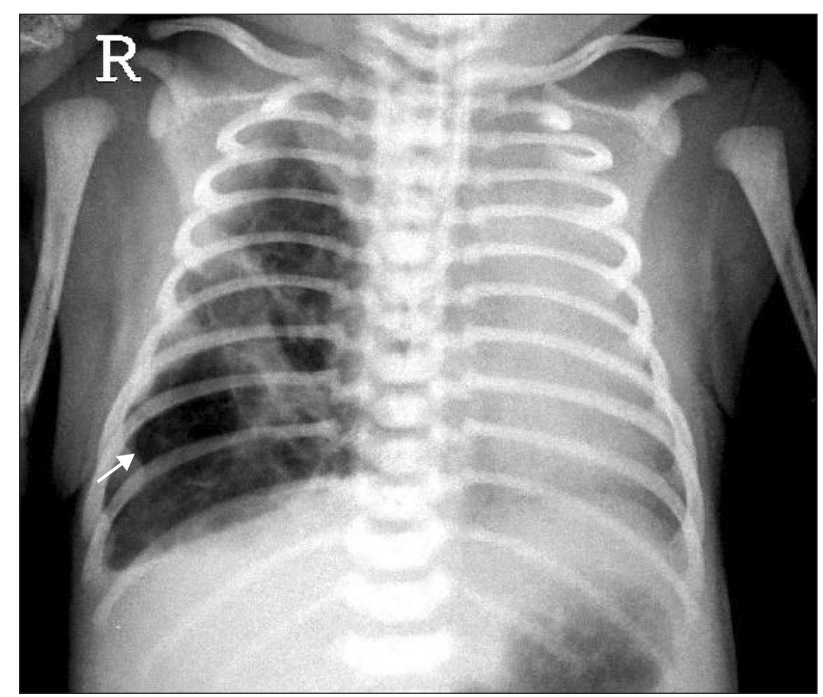

Fig. 1. Chest X-ray of the neonate on first day of admission showing multiple cysts and hyperaeration of the right lower lobe with a mediastinal shift to the left (Arrow).
Olympus $^{\circledR}$, Japan) and a 5 Fr AEB $(2.5 \mathrm{~mm}$, shaft $1.7 \mathrm{~mm})$ were prepared for OLV, and it was planned to mount the AEB inside the ID $5.0 \mathrm{~mm}$ ETT under the guidance of FOB.

In the preoperative laboratory tests, hemoglobin was $17.6 \mathrm{~g} /$ $\mathrm{dL}$ and hematocrit was $47.8 \%$, while arterial blood gas analysis (ABGA) results were $\mathrm{pH} 7.355, \mathrm{PaCO}_{2} 44.6 \mathrm{mmHG}, \mathrm{PaO}_{2}$ $72.9 \mathrm{mmHg}$, and $\mathrm{O}_{2}$ saturation $94.0 \%$. The neonate entered the operating room in an intubated state and ventilating through an AMBU (air mask bag unit) bag with $5 \mathrm{~L} / \mathrm{min}$ oxygen. After transferring the neonate to the operating table, the ETT and the breathing circuit were connected immediately to begin manual ventilation, administering $5 \mathrm{~L} / \mathrm{min}$ of oxygen. The assistant simultaneously monitored the patient monitoring equipment: pulse oximetry, electrocardiography, non-invasive blood pressure (NIBP), and end-tidal $\mathrm{CO}_{2}\left(\mathrm{EtCO}_{2}\right)$. The vital signs at that point were oxygen saturation $92 \%$, blood pressure $52 / 33$ $\mathrm{mmHg}$, heart rate 140 beats per minute, and $\mathrm{EtCO}_{2} 42 \mathrm{mmHg}$.

Induction of anesthesia was conducted by peripheral intravenous administration of ketamine $7 \mathrm{mg}$, rocuronium bromide $2 \mathrm{mg}$, atropine $0.1 \mathrm{mg}$, and dexamethasone $0.5 \mathrm{mg}$. After loss of consciousness, controlled ventilation was begun using the pressure-controlled ventilation (PCV) mode with a respiration rate of 50 breaths per minute. After controlled ventilation with $100 \%$ oxygen for approximately 5 minutes, it was confirmed that oxygen saturation was consistently $100 \%$ for more than 3 minutes. Following suction of the intraoral secretion, extubation of the previously intubated ID $4.5 \mathrm{~mm}$ ETT was performed. Intubation with the ID $5.0 \mathrm{~mm}$ ETT using a curved blade laryngoscope was attempted, but the tracheal

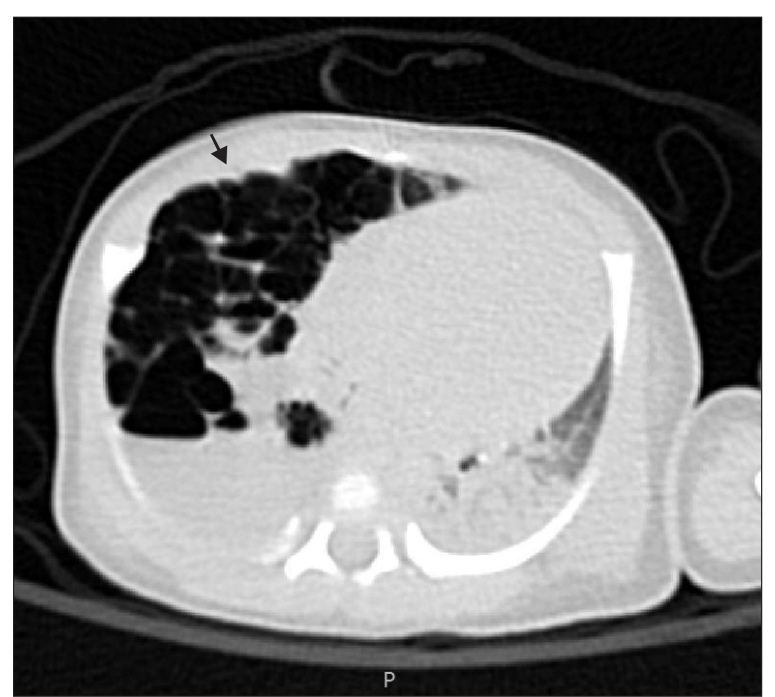

Fig. 2. CT scan showing multiple cyst like lesions with overexpansion in right lower lobe. Mediastinal shift to the left and right pleural effusion are noted. Also, multiple consolidations in right upper lobe and left lung is noted as well (Arrow). 
diameter of the neonate was too narrow and entry was not possible. Over the span of approximately 20 seconds, oxygen saturation rapidly declined from $100 \%$ to $60 \%$ and the heart rate fell from $130-140$ to 80 beats per minute; thus, mask ventilation was performed for approximately 3 minutes. The heart rate was restored to 150 beats per minute, but oxygen saturation did not rise to more than approximately $88-90 \%$. Hence, controlled ventilation was performed through intubation of ID $4.0 \mathrm{~mm}$ ETT, and then oxygen saturation increased and was maintained at $100 \%$. Here, mechanical ventilation was performed at the tidal volume of $30 \mathrm{ml}$, the respiration rate of 40 breaths per minute, the I : E ratio of $1.0: 1.5$, and the peak pressure was confirmed to be around 14-15 mmHg. After a while, the AEB was mounted inside the ETT and controlled ventilation was continuously maintained through the multiport airway adapter. To place the ETT in the right main bronchus, it was inserted to the point where the respiration sound of the left lung was weakest in auscultation, and the distance between end of the tube and the lip was approximately $12 \mathrm{~cm}$. The AEB was inserted to this point, and the ETT was retreated such that the distance between end of the tube and lips was $9.5 \mathrm{~cm}$ and then was fastened by turning the blocker port. The guide wire of the AEB was removed, and the right lung was isolated immediately by expanding the balloon with $0.5 \mathrm{ml}$ of air. Through auscultation, it was confirmed that there was no sound from the right lung and respiration was being performed in the left lung. While mounting the ETT to the right main bronchus, the oxygen saturation of the neonate declined to $80 \%$ but then was maintained at $98-100 \%$ after performing OLV.

Maintenance of anesthesia was achieved with 2.5 vol\% sevoflurane, ketamine, and fentanyl infusion. Before the operation, radial artery catheterization was attempted for direct measurement of arterial pressure and arterial blood gas analysis, but it was unsuccessful. Thus, monitoring was continued with the existing monitoring equipment. After starting OLV, oxygen saturation was maintained at $100 \%$, so $\mathrm{FiO}_{2}$ was lowered in phases to 0.5 and oxygen ventilation continued to be maintained at $98-100 \%$. Also during OLV, mechanical ventilation was performed at a respiration rate of 40 breaths per minute, the I : E ratio was $1.0: 1.5$, and the peak pressure was 15 $\mathrm{mmHg}$ (tidal volume $30 \mathrm{ml}$ ). Thus, there were no changes in pressure compared with before OLV. A thoracic right lower lobectomy was performed, and for the discharge of intracystic secretion, a $10 \mathrm{ml}$ syringe was connected to the lumen of the AEB. Intermittent negative pressure aspiration was performed 4-5 times to suction a total $1 \mathrm{ml}$ of yellowish serous fluid. The operation took 2 hours and 30 minutes, and OLV was continued for about 2 hours. During this time, the AEB was maintained in the mounted location without movement, and the operation was performed smoothly as there was a clear field of vision for operation. Bleeding during the operation was about $10 \mathrm{ml}$, vital signs were stable, and oxygen saturation was maintained successfully at $98-100 \%$ without symptoms of hypoxemia.

After completion of anesthesia, fentanyl $5 \mu \mathrm{g}$ was administered to maintain sedation. Then the AEB was removed and, with the ETT intact, the neonate was moved into the incubator for transport. During transfer to the neonatal intensive care unit, AMBU bag ventilation was performed through the intubated ETT, and oxygen saturation was continuously monitored. Laboratory tests performed directly after the operation were hemoglobin $14.1 \mathrm{~g} / \mathrm{dl}$ and hematocrit $37.4 \%$, while the ABGA results showed $\mathrm{pH}$ 7.494, $\mathrm{PaCO}_{2} 30.3$ mmHg, $\mathrm{PaO}_{2} 144.8$ $\mathrm{mmHg}$, and $\mathrm{O}_{2}$ saturation $99.1 \%$. The neonate was mechanically ventilated with HFOV in the neonatal intensive care unit (NICU) and switched to SIMV afterwards. One day post-operation, extubation of the ETT was performed. After extubation and without oxygen administration, the $A B G A$ results of the neonate showed $\mathrm{pH}$ 7.494, $\mathrm{PaCO}_{2} 32.5$ mmHg, $\mathrm{PaO}_{2} 84.2$ $\mathrm{mmHg}$, and $\mathrm{O}_{2}$ saturation $97.2 \%$. The neonate was discharged 8 days after the operation without specific complications.

\section{Discussion}

When a CCAM patient is undergoing lobectomy, OLV is usually performed. By performing OLV, the inflow of blood or infectious material from the affected lung into normal lung tissue can be prevented. Also, positive pressure ventilation solely by the unaffected normal lung becomes possible, and the wider field of vision makes the surgical approach more convenient [2].

Although various methods of OLV exist, it is more difficult to perform in neonates and infants than in adults due to the narrow trachea. A method that can be attempted easily without any specific technique is deliberate endobronchial intubation of a single-lumen ETT into the bronchus. The double-lumen tube (DLT) [3] and univent tube ${ }^{\circledR}$ [7] have relatively large outer diameters, so it is difficult to use them in infants younger than 6 years. A case was reported in which a Marraro bilumen tube, a modified form of DLT, was used in an infant younger than 3 years and between $2.7-12 \mathrm{~kg}$ [8], but it is no longer utilized.

Bronchial blockers such as the Fogarty embolectomy catheter, Arrow balloon wedge catheter, and AEB are all methods commonly used for OLV in infants. Of these, AEB is different from the other bronchial blockers in that it was developed for OLV from the outset. Hence, it has a high-volume, low-pressure balloon that can reduce the possibility of damaging the soft tissue inside the bronchial tubes. In addition, a multiport airway adapter exists, which has the advantage that oxygen can be provided continuously while adjusting the position of the AEB. The 5 Fr AEB developed for infants can be inserted within the ID $4.5 \mathrm{~mm}$ ETT using a $2.0 \mathrm{~mm}$ FOB [4], and Stephenson and 
Seefelder [5] reported a method of performing OLV through extraluminal placement of the 5 Fr AEB in infants younger than 24 months.

In this case, it was decided to use the $5 \mathrm{Fr}$ AEB as a method for OLV in a 4-day-old neonate. Because precise OLV can also affect the results of the operation [9], the authors had several methods planned before the operation. Since the smallest FOB in our hospital was $3.1 \mathrm{~mm}$, inserting the AEB using the FOB was considered the optimal method. For this, it was decided to use an ETT of ID $5.0 \mathrm{~mm}$. According to the neonate's chest $\mathrm{CT}$, the diameter of the trachea directly below the glottis was measured to be $6.47 \mathrm{~mm}$, and the entry of the ID $5.0 \mathrm{~mm}$ ETT (OD $6.9 \mathrm{~mm}$ ) was thus anticipated to be difficult. However, as this is one of the most precise OLV methods, the decision was made to attempt it first. Given that the neonate was already intubated with an ETT of ID $4.5 \mathrm{~mm}$, it had to be changed to an ID $5.0 \mathrm{~mm}$ ETT in the operating room. As anticipated, the ID 5.0 $\mathrm{mm}$ ETT was unable to pass through the trachea, and during this process the oxygen saturation declined rapidly.

The second method that had been preplanned as an alternative was to insert the AEB first and then perform the intubation of an ID $3.5 \mathrm{~mm}$ ETT to position the AEB outside of the ETT and adjust the position of the AEB with FOB. However, because oxygen desaturation progressed more quickly than expected, this method could not be attempted as it seemed to be impossible to perform while maintaining oxygen saturation.

Therefore, it was decided to perform the third method, which was to deliberately insert the ID $4.0 \mathrm{~mm}$ ETT into the right bronchus to mount the AEB inside the ETT and then retreat the ETT back to the trachea. In this method, the size of the ETT should be decided by considering the diameter of the right bronchus. The ratio of the trachea and right bronchus in infants is approximately $1: 0.86$ [10]. In our case, an ID $4.5 \mathrm{~mm}$ ETT (OD $6.2 \mathrm{~mm}$ ) had been intubated comfortably in the neonate, and the anticipated diameter of right bronchus was about 5.56 $\mathrm{mm}$. Thus, an ETT of ID $4.0 \mathrm{~mm}$ (OD $5.6 \mathrm{~mm}$ ) was selected for intubation into the right bronchus. This method's shortcoming is that the position of the AEB cannot be accurately confirmed with FOB, but it can be performed more easily and quickly compared with the previously mentioned methods. Even when the AEB is not intubated in the correct location on the first attempt, the position can be readjusted while maintaining oxygen saturation through the multiport airway adaptor by ventilating with the ETT already intubated in the trachea. Thus, this was concluded to be the safest method for the neonate.

Sands et al. [11] analyzed the decline of oxygen saturation in premature infants according to apnea time, examining three groups in which the oxygen partial pressure was $100 \mathrm{mmHg}, 80$ $\mathrm{mmHg}$, and $60 \mathrm{mmHg}$, respectively. According to the results, when the oxygen partial pressure was $100 \mathrm{mmHg}$, apnea con- tinuing for approximately 10 seconds resulted in an oxygen saturation reduction from $98 \%$ to $80 \%$, and 20 seconds elapsing resulted in a decline of oxygen saturation to less than $60 \%$. When the oxygen partial pressure was $60 \mathrm{mmHg}$, it took less than 10 seconds for the oxygen saturation to decline from $90 \%$ to $60 \%$. Although this was an analysis of premature infants, it is thought that the results will not largely differ for full-term neonates with accompanying RDS. In our case, endotracheal intubation was attempted after confirming that oxygen saturation was maintained at $100 \%$ through controlled ventilation with $100 \%$ oxygen; however, the oxygen saturation declined rapidly from $100 \%$ to $60 \%$ during an apnea duration of less than 20 seconds. On the basis of these results, for neonates with RDS, it is very important in anesthesia management to maintain high initial oxygen partial pressure through sufficient preoxygenation before anesthesia and to perform endotracheal intubation quickly as possible to maintain the airway. Also, it is important to predict beforehand the time taken for progress of desaturation by considering the preoperative oxygen partial pressure of the neonate. Then, after simulation of various intubation methods, the methods that cannot succeed within the predicted time should not be attempted regardless of their accuracy and advantages. In our case, the authors had first attempted to extubate the inserted ID $4.5 \mathrm{~mm}$ ETT and intubate the ID $5.0 \mathrm{~mm}$ ETT, as accurate OLV was considered to be important. However, we experienced a rapid decline of oxygen saturation owing to the difficulty of intubation due to the narrow trachea of the neonate. As a result, it would have been more beneficial to the neonate to perform, from the outset, faster OLV using the $4.0 \mathrm{~mm}$ ETT, and it was unfortunate that we did not attempt to insert the already intubated $4.5 \mathrm{~mm}$ ETT into the right main bronchus. As the outer diameter of the $4.5 \mathrm{~mm}$ ETT is $6.2 \mathrm{~mm}$, it is slightly larger compared with the anticipated right main bronchus diameter of $5.56 \mathrm{~mm}$, but it was worth attempting the method of inserting the ETT as far as the entrance of the right main bronchus to insert the AEB. The main advantage of this method is that oxygen can be provided continuously to the neonate during the manipulation of OLV, as the ETT is not replaced.

In a report regarding OLV in CCAM neonates with RDS, Hugh and Cameron presented a case in which an awake intubation was successful using a 5 Fr AEB in a neonate with CCAM in the left lower lobe and accompanying right mediastinal shift [12]. The neonate in that case was a newborn of 10 days who had shown tachypnea after birth, which was treated with continuous positive airway pressure (CPAP) through a nasal cannula, maintaining respiration with low-flow oxygen. The authors maintained spontaneous respiration until OLV was achieved, as positive ventilation can worsen the mediastinal shift and cause cardiopulmonary collapse. Kumagai et al. [13] also 
attempted awake intubation on left lower lobe CCAM neonates with right mediastinal shift for the same reasons as above. In this case, severe oxygen desaturation developed unexpectedly as spontaneous respiration weakened due to sevoflurane. Hence, endotracheal intubation was performed immediately. However, because the oxygen saturation continued to decline even after controlled ventilation was performed through the ETT, the ETT was inserted farther into the right bronchus, and oxygen saturation ascended to $99 \%$ after OLV was achieved.

In neonates with cystic lung lesions such as CCAM, positive ventilation can expand the site of disease to cause various complications, and thus it can be helpful to slowly induce anesthesia while maintaining spontaneous respiration. However, a relatively long time is taken for success, and oxygen desaturation can occur as the spontaneous respiration of the neonate is weakened during inducement of the anesthesia. Thus, this approach should be selected in consideration of the state of the neonate before operation. Our case is different from the previously described cases in that the oxygen desaturation of the neonate had been improved by performing positive ventilation through SIMV in the NICU, and there were no observations of deterioration such as expansion of the cystic lesion. Also, RDS had progressed to the point where the neonate could not maintain oxygen saturation with spontaneous respiration, making awake intubation impossible, and the authors decided to intubate after anesthesia.

Similar to the two cases mentioned above, the oxygen satu- ration of the neonate improved after OLV. This is a different condition from the occurrence of deoxygenation due to ventilation/perfusion mismatch in general OLV or in infants with small CCAM lesions. The reason for this is that in neonate CCAM with RDS, the increase in dead space resulting from the pressure on the healthy lung by the affected site's expansion through positive pressure during general both-lung ventilation could be prevented by OLS. Also, by performing OLV, hypoxic pulmonary vasoconstriction develops in the affected lung, and the resulting reduction in the amount of blood shunted would have protected the arterial blood oxygen partial pressure. In this case, after OLV, intermittent negative pressure aspiration through AEB was performed several times to suction yellowish serous fluid and air. The fact that matter existing inside the lesions could be discharged with ease is thought to have contributed to the improvement of oxygen saturation during OLV, to a certain degree.

In conclusion, when performing OLV in neonates, various methods should be planned in consideration of the equipment in the hospital and technique of the anesthesiologist. Especially in neonates with accompanying RDS, a sharp decline in oxygen saturation can occur within short apnea episodes. Therefore, it is important to predict the time taken for oxygen desaturation beforehand to plan for a method that can succeed quickly and accurately within this time limit, as well as to perform the procedure in a state of sufficient preoxygenation before anesthesia.

\section{References}

1. Rothenberg SS. Thoracoscopic pulmonary surgery. Semin Pediatr Surg 2007; 16: 231-7.

2. Hammer GB. Pediatric thoracic anesthesia. Anesthesiol Clin North America 2002; 20: 153-80.

3. Hammer GB, Fitzmaurice BG, Brodsky JB. Methods for single-lung ventilation in pediatric patients. Anesth Analg 1999; 89: 1426-9.

4. Wald SH, Mahajan A, Kaplan MB, Atkinson JB. Experience with the Arndt paediatric bronchial blocker. Br J Anaesth 2005; 94: 92-4.

5. Stephenson LL, Seefelder C. Routine extraluminal use of the 5F Arndt Endobronchial Blocker for one-lung ventilation in children up to 24 months of age. J Cardiothorac Vasc Anesth 2011; 25: 683-6.

6. Hammer GB, Harrison TK, Vricella LA, Black MD, Krane EJ. Single lung ventilation in children using a new paediatric bronchial blocker. Paediatr Anaesth 2002; 12: 69-72.

7. Hammer GB, Brodsky JB, Redpath JH, Cannon WB. The Univent tube for single-lung ventilation in paediatric patients. Paediatr Anaesth 1998; 8: 55-7.

8. Pawar DK, Marraro GA. One lung ventilation in infants and children: experience with Marraro double lumen tube. Paediatr Anaesth 2005; 15: 204-8.

9. Guruswamy V, Roberts S, Arnold P, Potter F. Anaesthetic management of a neonate with congenital cyst adenoid malformation. Br J Anaesth 2005; 95: 240-2.

10. Altman PL, Dittmer DK. Respiratory and circulation. Michigan, Federation of American Society for Experimental Biology. 1971, pp 105-8.

11. Sands SA, Edwards BA, Kelly VJ, Davidson MR, Wilkinson MH, Berger PJ. A model analysis of arterial oxygen desaturation during apnea in preterm infants. PLoS Comput Biol 2009; 5: e1000588.

12. Hugh D, Cameron B. Anesthetic management of a neonate with a congenital cystic adenomatoid malformation and respiratory distress associated with gross mediastinal shift. Paediatr Anaesth 2009; 19: 272-4.

13. Kumagai K, Nishiwaki K, Sato K, Kitamura H, Yano K, Onishi S, et al. Unilateral pulmonary cystic enlargement in a newborn: remember the onesided blind intubation. Paediatr Anaesth 2000; 10: 111-3. 